4 Cooke RWI, Morgan MEI. Prophylactic ethamsylate for periventricular haemorrhage. Arch Dis Child 1984;59:82-3

L M S Dubowitz AND A Whitelaw Department of Paediatrics and Neonatal Medicine, Hammersmith Hospital,

Ducane Road, London WI2 OMS

Drs Cooke and Morgan comment:

Drs Dubowitz and Whitelaw raise objections to our paper on three fronts; that developmental assessment was not 'blind', that some of the control group were 'historical' controls, and that published data might be used for advertising purposes.

While accepting that it would be theoretically possible to know at follow up which treatment an infant had received, we feel that in practice such a bias probably did not occur. The infants are being followed up in a prospective study of nearly 500 survivors weighing under $1500 \mathrm{~g}$ and only a considerable feat of memory or extensive perusal of the intensive care notes would show the early management. Lack of staff prevents the luxury of a separate team to conduct our follow up studies.

The use of 'historical' controls for a part of the study was, we felt, better than no controls at all, although we accept the limitations on data interpretation imposed by their use. After our initial, double blind randomised controlled trial of ethamsylate we proposed a larger multicentred study on a similar basis, but were criticised by several colleagues (including some from the Hammersmith Hospital) for proceeding without a period of follow up to show that the drug itself produced no detectable problems later. Also, it was suggested (again by colleagues at the Hammersmith Hospital) that ethamsylate merely removed a marker (haemorrhage) of underlying brain injury, thus increasing the number of babies without bleeds who were abnormal at follow up. Our short paper was intended as an attempt to answer these questions. The summary merely states what was found and our discussion neither makes nor implies extravagant claims for the efficacy or otherwise of the drug.

The use that drug companies or the non-medical press make of published data is not-as Drs Dubowitz and Whitelaw well know-under the control of authors. Freedom of information and public debate is part of our way of life in this country. The price that we pay for this freedom is the occasional misleading or inaccurate use of that data. We have not publicly promoted our data, but we have been approached by parents asking why their babies were not being treated with vitamin $\mathrm{E}$ to prevent brain damage', as a neonatologist on breakfast television had suggested its use.

A multicentre, randomised double blind controlled trial of ethamsylate began last year and is currently in progress. We would be pleased to provide information for other centres who might like to participate.

\section{Age as a main determinant of renal functional damage in urinary tract infection}

Sir,

Berg and Johansson present a study of 61 girls with recurrent urinary tract infections and at least one febrile infection ${ }^{1}$ in order to detect those patients at high risk of developing renal damage', and recommend early diagnosis 'to prevent future renal damage'. This study neither aids detection of those at risk, nor presents evidence that early diagnosis was also the outcome.

Firstly, they do not state how their patients were selected. If the criteria for entering the study were in any way age dependent, they could make no comparison across age groups. Secondly, they present a single estimation of glomerular filtration rate and then discuss the period when deterioration occurred without any sequential data. Two examples illustrate why this is insufficient. It may be that some children have dysplastic kidneys which are also vulnerable to infection, present early, and deteriorate no matter what treatment is instituted. Alternatively, suppose the chance of deteriorating renal function is independent of age and simply increases with the duration of follow up. The children presenting early would tend to have longer follow up from the first infection and greater chance of renal damage. Berg and Johansson, however, make no attempt to control for the duration of follow up, they simply state 'there was no sign of decreasing glomerular filtration rate with increasing follow up time', without presenting data on this vital point.

In summary, their data is difficult to interpret without more information.

\section{J Poulton \\ The Children's Hospital, Ladywood Middleway. Ladywood, \\ Birmingham B16 8ET}

Drs Berg and Johansson comment:

As stated in our paper, ${ }^{1}$ patients who had had signs of acute pyelonephritis (that is, fever and raised erythrocyte sedimentation rate) were selected for the study. Furthermore, as stated in the discussion section, indications for the renal function tests were abnormal intravenous urogram findings or frequent recurrences of urinary tract infection. Since renal function is not fully developed until the age of $1 \frac{1}{2}$ to 2 years, patients younger than that were not studied.

In our study we presented single glomerular filtration rate estimations only, but a paper presenting data from follow up investigations is in preparation. We agree that some patients might have had dysplastic kidneys but this diagnosis cannot be settled with certainty without renal biopsy or at necropsy. We believe that the three patients with very low glomerular filtration rates might have dysplastic kidneys.

Some information on glomerular filtration rates in relation to follow up time is available from Figs. $1-3$ of our article. In Fig. 1 the glomerular filtration rate was related to the age of the patient at the time of investigation. All patients with glomerular filtration rates less than -2 SD had their first pyelonephritis before the age of 3 years. If the three patients with the lowest rates are excluded, there is no tendency to decreasing renal function with age or follow up time (age, 1 to 3 years). To clarify this question further we now include a Figure in which the glomerular filtration rate is related to the duration of follow up. As mentioned in the paper, ${ }^{1}$ the mean follow up 